\title{
Improved Maximum Likelihood Estimation of Heston Model and Pricing Efficiency Test: Hong Kong Hang Seng Index Option
}

\author{
Huan Wang, ${ }^{1}$ Bin Song, ${ }^{2}$ and Dongmei Guo ${ }^{3}$ \\ ${ }^{1}$ Beijing Applied Information Institute of Technology, Beijing 100091, China \\ ${ }^{2}$ Investment Department, School of Management Science \& Engineering, Central University of Finance and Economics, \\ Beijing 100081, China \\ ${ }^{3}$ School of Economics, Central University of Finance and Economics, Beijing 100081, China
}

Correspondence should be addressed to Bin Song; selviasong@163.com

Received 20 April 2016; Accepted 12 July 2016

Academic Editor: Shaoyi He

Copyright (C) 2016 Huan Wang et al. This is an open access article distributed under the Creative Commons Attribution License, which permits unrestricted use, distribution, and reproduction in any medium, provided the original work is properly cited.

This paper selects improved maximum likelihood method to conduct parameter estimation of Heston model, and results show that the share option pricing performance of Hang Seng Index is better and pricing error of at-the-money options is the smallest. By comparing parameter estimation of samples in different intervals, it has been found that parameter estimated results of two-year market data are obviously inferior to estimated effect of one-year data.

\section{Introduction}

This paper adopts Maximum Likelihood Estimation (MLE) to estimate Heston model parameters and test pricing efficiency using Hang Seng Index Option data. Empirical results will be given for reference for promotion of China's stock index options, provide the scientific evidence for transaction pricing and hedging of issuers and investors, and lay a foundation for stable and healthy development of stock index options market.

Cox and Ross [1] elicited CEV (Constant Elasticity of Variance) options pricing model on the condition that variance of underlying asset price yield rate was proportional to share price. Then Hull and White [2] assuming variance of asset price was subject to Geometric Brownian motion showed that option price was equal to mean value of BlackScholes option prices of average variance of yield rates within duration of options. And Scott [3] and E. M. Stein and J. C. Stein [4] developed O-U (Ornstein-Uhlenbeck) process with mean reversion to describe variance of assets return. Then Heston [5] disproved assumption that risk premium of volatility and correlation coefficients were equal to zero based on O-U (Ornstein-Uhlenbeck) process. And Bates [6] brought random jumps into random volatility model and obtained analytic solution of European style option price through Fourier reverse transformation of characteristic equation complex value. Then the results were promoted by Bakshi et al. [7] by adding random interest rate. In another study, Carr and $\mathrm{Wu}[8]$ described asset price process using Levy Process with specified tail index and skewness parameter. However, many studies focus on Heston Model $[9,10]$; for example, Ahlip and Rutkowski employed Heston model to describe exchange rate changes and CIR interest rate model to describe interest rate changes and finally they obtained analytic solution; Leung adopted Heston model to solve analytic pricing problem of back-looking options of floating exercise price. Different from the assumption that volatility is subject to some function or process in the above models, implied volatility is volatility value deducted reversely by substituting market price of options into BlackScholes options pricing equation. For example, Dupire [11] put forward local volatility model based on implied volatility and reconstruct volatility of underlying asset in virtue of quoted price of Black-Scholes options and regularization method and then obtaining analytic formula of volatility.

Heston model has been widely applied to industries and academic fields; the reason is that the characteristic equation method is employed to obtain analytic solution of 
options price while improving the assumption of constant volatility, so it is a major breakthrough in researches on random volatility models. Since Heston model is a nonlinear and non-Gaussian state-dependent space defined and its likelihood function form is quite complicated, thus it makes it very difficult to estimate parameters of Heston model. But there have been some studies on parameter estimation of Heston model. For example, Basawa and Prakasa Rao [12] elicited a function of drift coefficients and diffusion coefficients about unknown parameters by nonlinear Kalman filtering and conducted a MLE on model parameters based on continuous observed values of unknown parameters. Jacquier et al. [13] conducted parameter estimation through Markov Chain Monte Carlo. Christoffersen and Jacobs [14] adopted nonlinear least squares method to conduct parameter estimation of random volatility models. Ruiz [15] first proposed Pseudo-Maximum Likelihood (QML) to estimate parameters of random volatility models for the first time. But Ait-Sahalia [16] developed an Approximate Maximum Likelihood (AML) function and tried to solve incompatible problem of estimated results caused by directly applying AML to disperse observation. And Aït-Sahalia and Kimmel [17] gathered price data of S\&P 500 Index Options market and used AML function to conduct parameter estimation of several random volatility models and obtained good estimation results. This paper, based on research of Ait-Sahalia, adopts MLE to conduct parameter estimation and empirical analysis.

\section{Heston Model}

In this section, we will briefly review Heston model.

Assume that Heston model satisfies the following conditions:

(1) Stock price follows stochastic process related to current volatility. (2) Short selling is allowed in stock market without transaction cost. (3) There is no stock dividend within the term of validity of options.

In Heston model, underlying asset price follows stochastic differential equation:

$$
d S=\mu S d t+\sqrt{Y} S d W_{S}
$$

where $S$ is underlying asset price, $Y$ is instantaneous variance of underlying asset price, and $d W_{S}$ is a Brownian movement. The variance $Y$ derived by

$$
d Y=k(\theta-Y) d t+\sigma \sqrt{Y} d W_{Y},
$$

where $\kappa$ is mean reversion parameter, $\theta$ is long-term volatility level, and $\sigma$ is volatility of $Y$. $W_{S}$ has correlation $\rho$ with $W_{Y}$ :

$$
d W_{S} d W_{Y}=\rho d t
$$

By using Ito Lemma and no arbitrage argument, Heston arrived at the following partial differential equation:

$$
\begin{aligned}
\frac{\partial C}{\partial t}+ & \frac{S^{2} Y}{2} \frac{\partial^{2} C}{\partial S^{2}}+\gamma S \frac{\partial C}{\partial S}-\gamma C+[k(\theta-V)-\lambda Y] \frac{\partial C}{\partial Y} \\
& +\frac{\sigma^{2} Y}{2} \frac{\partial^{2} C}{\partial Y^{2}}+\rho \sigma S Y \frac{\partial^{2} C}{\partial S \partial Y}=0 .
\end{aligned}
$$

$\lambda$ represents the price of volatility risk; Heston made an argument about analytic solution of the model that it had a form which is similar to Black-Scholes formula:

$$
C\left(S_{t}, Y_{t}, t, T\right)=S_{t} P_{1}-K e^{-r(T-t)} P_{2} .
$$

$P_{1}$ and $P_{2}$ are defined by the following Fourier Transform:

$$
\begin{array}{r}
P_{j}\left(x, Y_{t}, T, K\right) \\
=\frac{1}{2}+\frac{1}{\pi} \int_{0}^{\infty} \operatorname{Re}\left(\frac{e^{-i \phi \ln (K)} f_{j}\left(x, Y_{t}, T, \phi\right)}{i \phi}\right) d \phi, \\
j=1,2, x=\ln \left(S_{t}\right),
\end{array}
$$

where

$$
\begin{aligned}
f_{j}\left(x, Y_{t}, T, \phi\right)= & \exp \left\{C(T-t, \phi)+D(T-t, \phi) Y_{t}+i \phi x\right\}, \\
C(T-t, \phi)= & \gamma \phi i \gamma \\
& +\frac{a}{\sigma^{2}}\left[\left(b_{j}-\rho \sigma \phi i+d\right) \gamma-2 \ln \left(\frac{1-g e^{d r}}{1-g}\right)\right], \\
D(T-t, \phi)= & \frac{b_{j}-\rho \sigma \phi i+d}{\sigma^{2}}\left(\frac{1-e^{d r}}{1-g e^{d r}}\right), \\
g= & \frac{b_{j}-\rho \sigma \phi i+d}{b_{j}-\rho \sigma \phi i-d}, \\
d= & \sqrt{\left(b_{j}-\rho \sigma \phi i\right)^{2}-\sigma^{2}\left(2 \mu_{j} \phi i-\phi^{2}\right)}, \\
j=1,2, \mu_{1}= & \frac{1}{2}, \mu_{2}=\frac{1}{2}, a=k \theta, b_{1}=k+\lambda-\rho \sigma, b_{2}=k+\lambda .
\end{aligned}
$$

\section{The Estimation Method}

In the paper, we choose maximum likelihood method to estimate Heston model parameters. First, we determine a likelihood function for the state variables, representing conditional probability density of state variables. Based on the closed form approximation method of Ait-Sahalia [16], we calculate transferring density likelihood function of state variables and obtained likelihood function of its conditional probability density according to Markov property of state variable, making MLE method be applied to parameter estimation of models in a simpler way.

3.1. Likelihood Function of Multivariate Diffusion Model. We present the equation in full generality:

$$
d X_{t}=\mu\left(X_{t} ; \theta\right) d t+\sigma\left(X_{t} ; \theta\right) d W_{t}
$$

where $X_{t}$ is an $m$-vector of state variables and $\mu\left(X_{t}, \theta\right)$ is an $m$-dimensional function, $\sigma\left(X_{t}, \theta\right)$ is $m \times m$ matrixvalued function, $W t$ is an $m$-dimensional canonical Brownian motion, and $\theta$ is model parameter. Equation (8) can express multivariate diffusion models, including relevancy between variables in $X_{t}$, like Heston model with correlation coefficient $\rho$ between two Brownian movements. The first element in $X_{t}$ usually represents underlying asset price $S_{t}, X_{t}$ can be expressed as $X_{t}=\left[S_{t} ; Y_{t}\right]^{\prime}$, and $Y_{t}$ is $(m-1)$-dimensional 
vector. On the condition that $X_{t}=x_{0}$, conditional probability density of $X_{t+\Delta}=x$ will be called transfer equation, and it can be expressed as $p_{X}\left(x \mid x_{0}, \Delta\right)$. As we suppose that discrete data is of Markov characteristics, according to Bayes rules, simple form of log-likelihood function can be easily obtained:

$$
\ell_{n}(\theta, \Delta)=\sum_{i=1}^{n} l_{X}\left(X_{i \Delta} \mid X_{(i-1) \Delta}, \Delta\right)
$$

where $l_{X}=\ln p_{X}$. Since analytic formula of $p_{X}$ and $\ln p_{X}$ cannot be obtained, then Ait-Sahalia [16] offered the analytic form of log-likelihood function of multivariate diffusion with the form of a power series:

$$
\begin{aligned}
l_{X}^{(J)}\left(\Delta, x \mid x_{0} ; \theta\right)= & -\frac{m}{2} \ln (2 \pi \Delta)-D_{v}(x, \theta) \\
& +\frac{C_{X}^{(-1)}\left(x \mid x_{0} ; \theta\right)}{\Delta} \\
& +\sum_{k=0}^{J} C_{X}^{(k)}\left(x \mid x_{0} ; \theta\right) \frac{\Delta^{k}}{k !},
\end{aligned}
$$

where $D_{v}(x ; \theta)=(1 / 2) \ln (\operatorname{det}[v(x ; \theta)])$ and $v(x)=\sigma(x) \sigma^{\prime}(x)$. This equation can be calculated to arbitrary order $J . C_{X}^{(k)}$ is corresponding to Taylor expansion nearby $x_{0}$ at order $j_{k}$. AitSahalia [16] indicates $j_{k}=2(J-k) . C_{X}^{(k)}$ calculated in this way will be expressed as $C_{X}^{\left(j_{k}, k\right)}$. Then (10) can be expressed as

$$
\begin{aligned}
\widetilde{l}_{X}^{(J)}\left(\Delta, x \mid x_{0} ; \theta\right)= & -\frac{m}{2} \ln (2 \pi \Delta)-D_{v}(x, \theta) \\
& +\frac{C_{X}^{\left(j_{-1},-1\right)}\left(x \mid x_{0} ; \theta\right)}{\Delta} \\
& +\sum_{k=0}^{J} C_{X}^{\left(j_{k}, k\right)}\left(x \mid x_{0} ; \theta\right) \frac{\Delta^{k}}{k !} .
\end{aligned}
$$

According to the method of Ait-Sahalia, we can use Kolmogorov equation to solve $C_{X}^{\left(j_{k}, k\right)}$.

3.2. Likelihood Function Calculation of Heston Model. Write Heston model in a multivariate model framework, and reexpress it as the following matrix form; consider it first under measure $Q$; then

$$
\begin{aligned}
d X_{t}= & d\left[\begin{array}{c}
S_{t} \\
Y_{t}
\end{array}\right] \\
= & {\left[\begin{array}{c}
(r-d) S_{t} \\
\kappa^{\prime}\left(\gamma^{\prime}-Y_{t}\right)
\end{array}\right] d_{t} } \\
& +\left[\begin{array}{cc}
\sqrt{\left(1-\rho^{2}\right) Y_{t}} S_{t} & \rho \sqrt{Y_{t}} S_{t} \\
0 & \sigma Y_{t}^{\beta}
\end{array}\right] d\left[\begin{array}{l}
W_{1}^{\mathrm{Q}}(t) \\
W_{2}^{\mathrm{Q}}(t)
\end{array}\right] .
\end{aligned}
$$

Then conduct logarithmetics of stock price to obtain the following form:

$$
\begin{aligned}
d\left[\begin{array}{l}
S_{t} \\
Y_{t}
\end{array}\right]= & {\left[\begin{array}{c}
r-d-\frac{1}{2} Y_{t} \\
\kappa^{\prime}\left(\gamma^{\prime}-Y_{t}\right)
\end{array}\right] d_{t} } \\
& +\left[\begin{array}{cc}
\sqrt{\left(1-\rho^{2}\right) Y_{t}} & \rho \sqrt{Y_{t}} \\
0 & \sigma Y_{t}^{\beta}
\end{array}\right] d\left[\begin{array}{l}
W_{1}^{\mathrm{Q}}(t) \\
W_{2}^{\mathrm{Q}}(t)
\end{array}\right] .
\end{aligned}
$$

Convert it under measure $P$; (12) will be transformed into

$$
\begin{aligned}
d\left[\begin{array}{c}
S_{t} \\
Y_{t}
\end{array}\right]= & {\left[\begin{array}{c}
r-d-\frac{1}{2} Y_{t}+b Y_{t} \\
\kappa\left(\gamma-Y_{t}\right)
\end{array}\right] d_{t} } \\
& +\left[\begin{array}{cc}
\sqrt{\left(1-\rho^{2}\right) Y_{t}} & \rho \sqrt{Y_{t}} \\
0 & \sigma Y_{t}^{\beta}
\end{array}\right] d\left[\begin{array}{l}
W_{1}^{P}(t) \\
W_{2}^{P}(t)
\end{array}\right], \\
a & =r-d, \\
b & =\lambda_{1}\left(1-\rho^{2}\right)+\lambda_{2} \rho-\frac{1}{2}, \\
\kappa & =\kappa^{\prime}-\lambda_{2} \sigma, \\
\gamma & =\left(\frac{\kappa+\lambda_{2} \sigma}{\kappa}\right) \gamma^{\prime} .
\end{aligned}
$$

Stock dividend is not considered; then Heston model under $P$ measure can be simplified as

$$
\begin{aligned}
d\left[\begin{array}{c}
S_{t} \\
Y_{t}
\end{array}\right]= & {\left[\begin{array}{cc}
r+\left[\lambda\left(1-\rho^{2}\right)-\frac{1}{2}\right. \\
\kappa\left(\gamma-Y_{t}\right)
\end{array}\right] d_{t} } \\
& +\left[\begin{array}{cc}
\sqrt{\left(1-\rho^{2}\right) Y_{t}} & \rho \sqrt{Y_{t}} \\
0 & \sigma Y_{t}^{\beta}
\end{array}\right] d\left[\begin{array}{l}
W_{1}^{P}(t) \\
W_{2}^{P}(t)
\end{array}\right] .
\end{aligned}
$$

According to this model and the method about likelihood function analytic formula proposed by Ait-Sahalia [16], we can calculate coefficient $C_{X}^{\left(j_{k}, k\right)}$ in likelihood function of conditional probability density of state variables in Heston model. In Heston model, likelihood function of conditional probability density of state variable $X_{t}$ is

$$
\begin{aligned}
l_{X}^{(J)}\left(\Delta, x \mid x_{0} ; \theta\right)= & -\frac{m}{2} \ln (2 \pi \Delta)-D_{v}(x, \theta) \\
& +\frac{C_{X}^{(-1)}\left(x \mid x_{0} ; \theta\right)}{\Delta} \\
& +\sum_{k=0}^{J} C_{X}^{(k)}\left(x \mid x_{0} ; \theta\right) \frac{\Delta^{k}}{k !} .
\end{aligned}
$$

Using this likelihood expression formula, we input the observed value $\left[S_{t}, Y_{t}\right]$ and adopt Matlab to compile MLE function to estimate the parameters. 
3.3. Input Variable in the Likelihood Function. In the following, we divide two steps to talk about the input variable in the likelihood function. First, reduce spatial dimension of input variable; second, determine the replacement of momentary volatility.

(1) Reduce Spatial Dimension of Input Variable. We examine each of those steps to reduce spatial dimension of input variables. Firstly, we limit options duration $T_{i}-t$ to a fixed value to reduce a dimension. For example, the options are selected which will be due three months later among listed options on each trading day; secondly, we consider selecting a fixed option value which can be expressed as $S / K$. For a call option, in-the-money options refer to options with $S / K$ being greater than 1, at-the-money options refer to options with $S / K$ being 1, and out-of-the-money options refer to options with $S / K$ being less than 1 . Fixed exercise prices $K$ are easy way to reducing a dimension. However, set an exercise price of at-the-money option around share price or stock index in each trading day, and with the fluctuation of stock price $S$, the distribution of exercise price $K$ is not machine-parsable, so it cannot be guaranteed that option contract of exercise price $K$ exists in each trading day within sampling period when data is being collected. Based on this listing method, it is more feasible for us to set an option price of rational value state; thirdly, assume the option price is homogeneous. Payoff of European call option is a linear function about stock price and exercise price. The price of one European call option can be expressed as $\left[E\left(S_{T}\right)-K\right]^{+} \cdot e^{-\int r(t) d t}$, so it can be considered that

$$
\begin{aligned}
C\left(T, \alpha \mathrm{S}_{T}, \alpha K\right) & =\left(\alpha S_{T}-\alpha K\right)^{+}=\alpha\left(\mathrm{S}_{T}-K\right)^{+} \\
& =\alpha C\left(T, \mathrm{~S}_{T}, K\right) .
\end{aligned}
$$

Generally, the price of one option is not of homogeneity except for some additional limitations for the model. For example,

$$
\begin{aligned}
\sigma_{1}\left(X_{t}\right) \sigma_{1}^{\prime}\left(X_{t}\right) & =\varphi_{11}\left(Y_{t}\right) S_{t}^{2}, \\
\sigma_{1}\left(X_{t}\right) \sigma_{i}^{\prime}\left(X_{t}\right) & =\varphi_{1 i}\left(Y_{t}\right) S_{t}=\varphi_{i 1}\left(Y_{t}\right) S_{t}, \quad i>1 \\
\sigma_{i}\left(X_{t}\right) \sigma_{j}^{\prime}\left(X_{t}\right) & =\varphi_{i j}\left(Y_{t}\right), \quad i>1, j>1 \\
\mu_{i}^{\mathrm{Q}}\left(X_{t}\right) & =\psi_{i}\left(Y_{t}\right), \quad i>1 .
\end{aligned}
$$

So far, we have successfully reduced three dimensions of input variables.

(2) Replacement of Instantaneous Volatility $Y_{t}$. The instantaneous volatility $Y_{t}$ of stock price is an unobservable variable. Generally there are two methods to solve this problem.

One method is to modify input variables. Convert input variables of likelihood function from observable variables into unobservable variables.

As $Y_{t}$ is unobservable, we convert it into an observable variable, such as $C_{t}$. We have obtained expression formula of conditional probability density log-likelihood function (16) adopted in parameter estimation. This expression formula requires input variable to be $\left[S_{t} ; Y_{t}\right]^{\prime}$. According to
MLE, a specific observed value is input through all forms of parameter combination $\theta$. However, during the process of searching the optimal $\theta$, with continuous variation of $\theta, X_{t}$ value will change, which indicates that, in a strict sense, the parameter estimation method which inputs $\left[S_{t} ; Y_{t}\right]$ to conduct maximum searching of likelihood function is unscientific.

Hence, we can try to convert input variable from unobservable state variable $X_{t}=\left[S_{t} ; Y_{t}\right]^{\prime}$ into observable asset price vector $G_{t}=\left[S_{t} ; C_{t}\right]^{\prime}$, thus converting input variable $\left[S_{t} ; Y_{t}\right]^{\prime}$ into $\left[S_{t} ; C_{t}\right]^{\prime}$. In fact, what we obtain from the preceding part of this paper is likelihood function about $X_{t}=$ $\left[S_{t} ; Y_{t}\right]^{\prime}$. A series of volatility $Y_{t}$ can be used to calculate a series of option price for one set of parameters. By comparing it with $C_{t}$, select the most appropriate volatility $Y_{t}$ of this set of parameters, so there will be corresponding $X_{t}=\left[S_{t} ; Y_{t}\right]^{\prime}$ for this set of parameters.

In actual operation of parameter estimation, it is necessary to make certain adjustment of likelihood function about $X_{t}$. Then $X_{t}$ needs to be multiplied by a Jacobian term, and it will be a partial differential equation of state variable $X_{t}$ with respect to $S_{t}$ and $C_{t}$. Put these differential expressions in a matrix, and Jacobian term is the determinant of this matrix, which is in the following form:

$$
\begin{aligned}
& J_{t}=\operatorname{det}\left[\begin{array}{cccc}
\frac{\partial S_{t}}{\partial S_{t}} & \frac{\partial S_{t}}{\partial Y_{t}(1)} & \cdots & \frac{\partial S_{t}}{\partial Y_{t}(N)} \\
\frac{\partial C_{t}(1)}{\partial S_{t}} & \frac{\partial C_{t}(1)}{\partial Y_{t}(1)} & \cdots & \frac{\partial C_{t}(1)}{\partial Y_{t}(N)} \\
\vdots & \vdots & \ddots & \vdots \\
\frac{\partial C_{t}(N)}{\partial S_{t}} & \frac{\partial C_{t}(N)}{\partial Y_{t}(1)} & \cdots & \frac{\partial C_{t}(N)}{\partial Y_{t}(N)}
\end{array}\right] \\
& =\operatorname{det}\left[\begin{array}{cccc}
1 & 0 & \cdots & 0 \\
\frac{\partial C_{t}(1)}{\partial S_{t}} & \frac{\partial C_{t}(1)}{\partial Y_{t}(1)} & \cdots & \frac{\partial C_{t}(1)}{\partial Y_{t}(N)} \\
\vdots & \vdots & \ddots & \vdots \\
\frac{\partial C_{t}(N)}{\partial S_{t}} & \frac{\partial C_{t}(N)}{\partial Y_{t}(1)} & \cdots & \frac{\partial C_{t}(N)}{\partial Y_{t}(N)}
\end{array}\right] \\
& =\operatorname{det}\left[\begin{array}{ccc}
\frac{\partial C_{t}(1)}{\partial Y_{t}(1)} & \cdots & \frac{\partial C_{t}(1)}{\partial Y_{t}(N)} \\
\vdots & \ddots & \vdots \\
\frac{\partial C_{t}(N)}{\partial Y_{t}(1)} & \cdots & \frac{\partial C_{t}(N)}{\partial Y_{t}(N)}
\end{array}\right] \text {. }
\end{aligned}
$$

The following is the process of converting likelihood function of state variable $X_{t}$ into that of state variable $G_{t}$ through Jacobian term. Suppose that transition density function of $X_{t}$ is $p_{X}\left(\Delta, x \mid x_{0} ; \theta\right)$ and that of $G_{t}$ is $p_{G}(\Delta, g \mid$ $\left.g_{0} ; \theta\right)$; write observable asset price vector $G_{t}$ as function of state variable $X_{t}$ :

$$
G_{t+\Delta}=f\left(X_{t+\Delta} ; \theta\right) .
$$


Then its inverse function to expresses that state is the function of observed asset prices:

$$
X_{t+\Delta}=f^{-1}\left(G_{t+\Delta} ; \theta\right) .
$$

Given that $G_{t}=g_{0}$, conditional probability density of $G_{t+\Delta}=g$ is

$$
\begin{aligned}
p_{G}\left(\Delta, g \mid g_{0} ; \theta\right)=\operatorname{det}\left(\frac{\partial f\left(f^{-1}(g ; \theta)\right)}{\partial x}\right)^{-1} \\
\cdot p_{X}\left(\Delta, f^{-1}(g ; \theta) \mid f^{-1}\left(g_{0} ; \theta\right) ; \theta\right) \\
=J_{t}\left(\Delta, g \mid g_{0} ; \theta\right)^{-1} \\
\cdot p_{X}\left(\Delta, f^{-1}(g ; \theta) \mid f^{-1}\left(g_{0} ; \theta\right) ; \theta\right),
\end{aligned}
$$

where the multiplier $J_{t}\left(\Delta, g \mid g_{0} ; \theta\right)$ will be calculated by referring to (19).

Observable asset prices have the property of Markov, and they can be obtained using Bayes Rule. Log-likelihood function for discrete data of vector $g_{t}$ at dates $t_{0}, t_{1}, \ldots, t_{n}$ can be expressed as

$$
\ell_{n}(\theta) \equiv n^{-1} \sum_{i=1}^{n} l_{G}\left(t_{i}-t_{i-1}, g_{t i} \mid g_{t i-1} ; \theta\right)
$$

where

$$
\begin{aligned}
l_{G}(\Delta, & \left.g \mid g_{0} ; \theta\right)=\ln p_{G}\left(\Delta, g \mid g_{0} ; \theta\right) \\
= & \ln J_{t}\left(\Delta, g \mid g_{0} ; \theta\right) \\
& \quad+l_{X}\left(\Delta, f^{-1}(g, \theta) \mid f^{-1}\left(g_{0}, \theta\right) ; \theta\right) .
\end{aligned}
$$

Then log-likelihood function about observable asset prices can be obtained.

Next we introduce the alternative method to replace momentary volatility by Black-Scholes implied volatility.

Input variable of this method is $\left[S_{t}, \sigma_{t}^{\mathrm{imvol}}\right]$. The evolution of stock price does not conform to the assumptions of Black-Scholes model in this paper; because of operability of empirical inspection, we will use implied volatility of short-term at-the-money options calculated by Black-Scholes formula as a substitute variable of momentary volatility of stock price. This method has greatly simplified complexity of parameter estimation, especially calculating Jacobian term according to when input variables are converted.

Here, the reasons for selecting implied volatility of shortterm at-the-money options to replace momentary volatility are as follows: firstly, based on preceding researches, as options are close to due, implied volatility of short-term atthe-money options is converged to momentary volatility of stock price; secondly, short-term at-the-money options are the most active option kind with the best mobility, so price of this kind of options will be less influenced by factors such as market microstructure; thirdly, compared with other options, prices of at-the-money options are the most sensitive to change of volatility, and a slight change of volatility will result in a major change of at-the-money options price. Correspondingly, if the observation error on options price is small, then the error of volatility will be less, which can be ignored.

There is one shortcoming of this method. It does not take some fluctuating factors under $Q$ measure. While in practical process of searching the best $\theta$, with continuous change of $\theta$, $X_{t}$ also changes not as invariable as input observable value $\left[S_{t}, \sigma_{t}^{\mathrm{imvol}}\right]$ is.

\section{Empirical Analysis: Based on Hong Kong Hang Seng Index Options}

On one hand, empirical analysis can examine applicability of Heston model to Hong Kong market, estimate relevant parameters, and provide investors of Hang Seng Index Options with a reference for price forecasting of future transactions. On the other hand, Hong Kong market is closed related with domestic market of China, so pricing method of Hong Kong market may have good reference significance for promoting Hushen 300 Index Options in China.

4.1. Data Selection. This section selects day-degree data of Hang Seng Index Options price in Hong Kong Exchange. The data set consists of continuous 500 transaction days spanning from November 24th, 2011, to November 29th, 2013, and complete 247 transaction days spanning from December 3rd, 2012, to November 29th, 2013.

In order to reduce dimensions to match number of parameters and input variables, we need to fix due dates of options selected in the sample. However, among option contract product sequences in Hong Kong Exchange, options that can be transacted each day include the following:

(1) Hang Seng Index Options expire at the end of each of the recent three month.

(2) Hang Seng Index Options expire on the last transaction day at the end of March, June, September, and December within the year.

Hence, it cannot be guaranteed that durations of options of each transaction day in the sample are identical. We can only defer the expiration date of sample options of each month by one month and guarantee that duration of selected option in the sample will be with limits. Specifically, we select data with its expiration date being 60-90 days. For example, for January 1st, we select the option contract that will expire at the end of March, so its duration will be about 90 days, but, for March 20th, we select the option contract which will become due at the end of May, so its duration will be about 70 days. For empirical research, we consider expiration dates of the two as identical.

For convenience, we only select call option contract among these data. Since the trading volume of in-themoney options of Hong Kong market is small, and some only have single-digit trading volume, thus we select atthe-money options and some out-of-the-money options to conduct an empirical analysis with big trading volumes. Based on variation situation of everyday Hang Seng Index, 
TABLE 1: Heston model estimation values and standard deviation of at-the-money call option and two out-of-the-money options based on three-month duration.

\begin{tabular}{lcccccc}
\hline $\begin{array}{l}\text { Heston model } \\
\text { parameters }\end{array}$ & $\begin{array}{c}\left(S_{t} / K\right)=1 \\
\text { Parameter } \\
\text { estimation values }\end{array}$ & $\begin{array}{c}\left(S_{t} / K\right)=1 \\
\text { Standard } \\
\text { deviation }\end{array}$ & $\begin{array}{c}\left(S_{t} / K\right)=0.95 \\
\text { Parameter } \\
\text { estimation value }\end{array}$ & $\begin{array}{c}\left(S_{t} / K\right)=0.95 \\
\text { Standard } \\
\text { deviation }\end{array}$ & $\begin{array}{c}\left(S_{t} / K\right)=0.92 \\
\text { Parameter } \\
\text { estimation value }\end{array}$ & $\begin{array}{c}\left(S_{t} / K\right)=0.92 \\
\text { Standard } \\
\text { deviation }\end{array}$ \\
\hline$\kappa$ & 0.4264 & 0.2784 & 0.4373 & 0.2086 & 0.4459 & 0.2113 \\
$\gamma$ & 0.0946 & 0.0181 & 0.0915 & 0.0163 & 0.0909 & 0.0168 \\
$\rho$ & -0.6284 & 0.0867 & -0.7011 & 0.0715 & -0.6812 & 0.0790 \\
$\lambda$ & 3.4543 & 3.0332 & 3.4280 & 2.9312 & 3.3788 & 2.7003 \\
$\sigma$ & 0.4822 & 0.0433 & 0.3565 & 0.0333 & 0.3421 & 0.0315 \\
\hline
\end{tabular}

TABLE 2: Parameter estimation results of 500-day at-the-money option price data.

\begin{tabular}{|c|c|c|c|c|c|}
\hline Heston model parameters & $\kappa$ & $\gamma$ & $\rho$ & $\lambda$ & $\sigma$ \\
\hline Estimated values of Heston model parameters & 1.1409 & 12.8701 & -0.9957 & -175.9290 & 0.0000 \\
\hline
\end{tabular}

we respectively find out exercise price $K$ of corresponding contracts approximate to at-the-money option $S_{t} / K \approx 1$, outof-the-money option $S_{t} / K \approx 0.95$, and $S_{t} / K \approx 0.92$ as well as closing price $C_{t}$ of these option contracts.

\subsection{Analysis of Empirical Results}

(1) Parameter Estimation Results and Analysis. Table 1 lists estimation results selecting at-the-money option price data and out-of-the-money option price data within the sample.

It can be seen from Table 1 that, among three samples, $\kappa$ obtained through the estimation basically stabilizes within $0.42-0.45$. Parameter $\kappa$ is mean reversion speed of volatility square which represents the speed of volatility and its square reversing to mean value when they deviate from the longterm mean value. Aït-Sahalia and Kimmel [17] conducted an empirical analysis using S\&P 500 and its stock index option data and obtained that estimated value of reversion rate $\kappa$ is 5.07, which means that, compared with American market, the mean reversion rate of Hong Kong market is slower, which indicates that when volatility in Hong Kong stock market deviates from mean value upwards or downwards, it will reverse to the mean level at a slow speed. Long-term mean value of volatility square is around 0.09 , which indicates that underlying asset price volatility will move around $30 \%$ within a long period, and correlation coefficient $\rho$ of two Brownian movements will be around -0.6 to -0.7 , and $\lambda$ stabilizes around 3.4 , but variable range estimated value of volatility $\sigma$ of stock index volatility is slightly larger, from 0.34 to 0.48 .

In the model, $\rho$ is the risk source of stock index risk source and stock index volatility square, that is, the correlation coefficient between two Brownian movements $d W_{1}$ and $d W_{2}$; the above empirical results show that $\rho$ is a negative value and remains within -0.6 to -0.7 , which indicates that HSI stock index yield rate has a negative correlation with its volatility. This result has overthrown complete negative correlation or complete noncorrelation between underlying asset price and its volatility in other random volatility models to exactly explained "leverage effect" observed in the market.
On the whole, standard deviation of estimated variable value of at-the-money option sample $\left(S_{t} / K\right)=1$ is higher than the two out-of-the-money option samples $\left(S_{t} / K\right)=0.95$ and $\left(S_{t} / K\right)=0.92$. We try to expand data length within the sample and select 500-day at-the-money option price data within the sample to conduct parameter estimation, and the results are as shown in Table 2.

It can be seen that, among 500-day parameter estimation results, interest rate $r$ has negative values, $\sigma$ is 0 , and reversed mean $\gamma$ of volatility mean reversion equation reaches as high as 12.8701 , which severely does not conform to current situation. This phenomenon can be explained from the angle of parameter estimation method. MLE method adopts closing price of at-the-money call option contracts of the current day as data of this day, so the bigger the selected sample is, the bigger the time span will be. If 500-day data in the sample is selected, sample span should be more than two years. As options market is not fixed, so when MLE method is used to conduct parameter estimation of options pricing research, larger sample size does not mean more ideal results. Instead, a proper sample size should be selected to guarantee adequacy of sample data and avoid estimation deviation caused by changes of multiperiod price characteristics.

(2) Analysis of Options Pricing Error. Here, according to parameter estimation results of data in option sample under different value states, we calculate options pricing errors 15 days beyond the sample, and Figures 1-3, respectively, express options pricing errors under different value states.

From Figures 1-3, we can see that it has a higher accuracy to estimate model parameters and deduce options price using at-the-money options, compared with the two out-of-the-money options, and pricing errors of 15 option contracts outside the sample are within $10 \%$, and there are 7 ones with their errors within $3 \%$ and 11 ones with errors within $6 \%$, which indicates that the model can be better priced at-the-money call options with expiration date being about 3 months. Table 3 reflects the average pricing error, where 


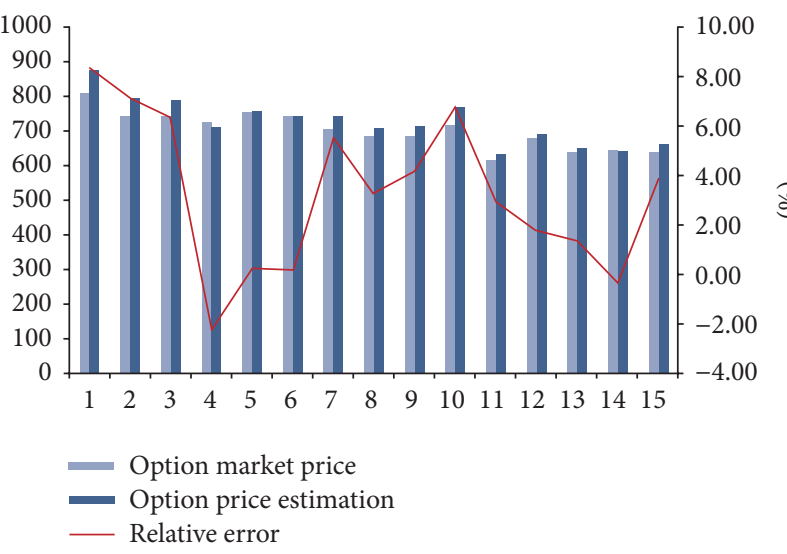

FIGURE 1: Options pricing error of at-the-money option $\left(\mathrm{S}_{t} / K\right)=1$ price.

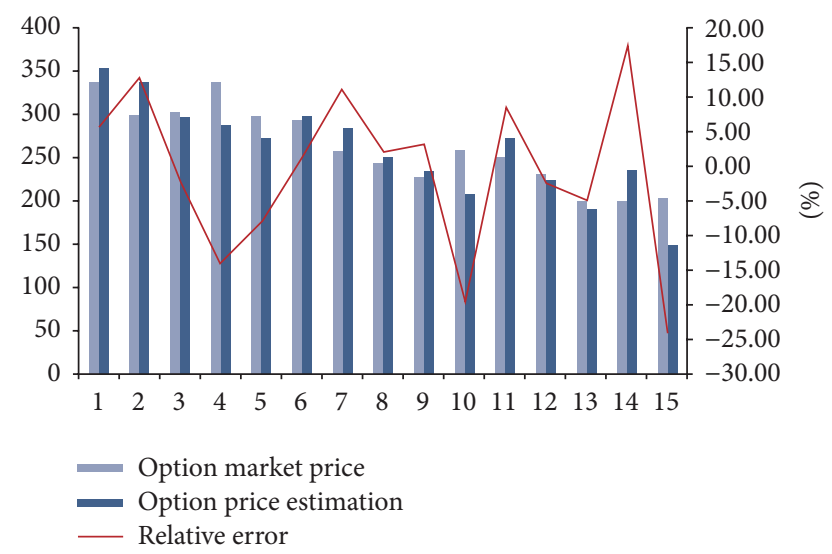

FIGURE 2: Options pricing error of out-of-the-money option $\left(\mathrm{S}_{t} / K\right)=0.95$ price.

TABLE 3: Options pricing error.

\begin{tabular}{lccc}
\hline & $\left(S_{t} / K\right)=1$ & $\left(S_{t} / K\right)=0.95$ & $\left(S_{t} / K\right)=0.92$ \\
\hline $\begin{array}{l}\text { Average absolute error } \\
\left(\bar{P}_{\text {absolute }}\right)\end{array}$ & $3.66 \%$ & $9.23 \%$ & $10.07 \%$ \\
$\begin{array}{l}\text { Average relative error } \\
\left(\bar{P}_{\text {actual }}\right)\end{array}$ & $3.30 \%$ & $-1.18 \%$ & $3.35 \%$ \\
\hline
\end{tabular}

$$
\begin{aligned}
\operatorname{Bias}_{i} & =C_{i}^{\text {model }}(t)-C_{i}^{\text {market }}(t), \\
\bar{P}_{\text {absolute }} & =\operatorname{average}\left(\sum_{t=1}^{T} \sum_{i=1}^{n(t)}\left|\frac{\operatorname{Bias}_{i}(t)}{C_{i}^{\text {market }}(t)}\right|\right), \\
\bar{P}_{\text {actual }} & =\operatorname{average}\left(\sum_{t=1}^{T} \sum_{i=1}^{n(t)} \frac{\operatorname{Bias}_{i}(t)}{C_{i}^{\text {market }}(t)}\right) .
\end{aligned}
$$

Other origins of parameter estimation error and model pricing error include simplification of real measure. In order to simplify the model and reduce number of parameters to be estimated, for the convenience of parameter estimation, the model adopted in the empirical analysis is the model form in risk neutral measure ( $Q$ measure); real measure ( $P$ measure) is not taken into consideration when parameters are being set,

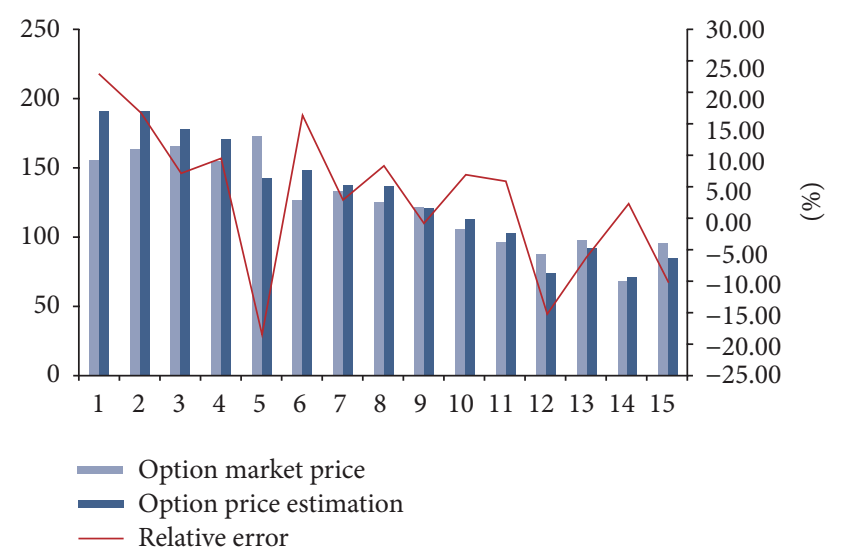

FIGURE 3: Options pricing error of in-the-money call option $\left(\mathrm{S}_{t} / K\right)=0.92$ price.

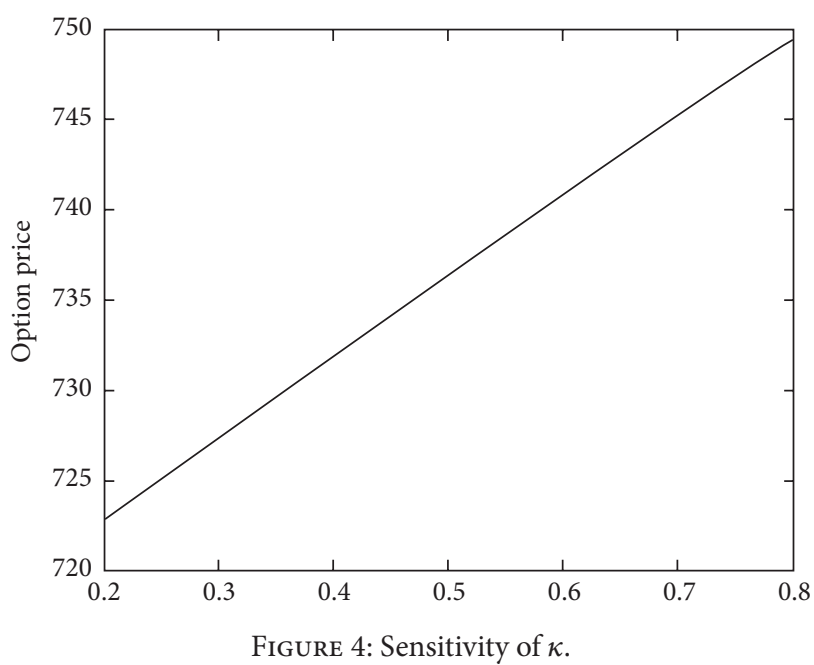

so price of risk factors in $P$ measure has not been estimated out; for example, Heston model in (17) is

$$
\begin{aligned}
d\left[\begin{array}{l}
S_{t} \\
Y_{t}
\end{array}\right]= & {\left[\begin{array}{c}
r-d-\frac{1}{2} Y_{t}+\lambda\left(1-\rho^{2}\right) Y_{t} \\
\kappa\left(\gamma-Y_{t}\right)
\end{array}\right] d_{t} } \\
& +\left[\begin{array}{cc}
\sqrt{\left(1-\rho^{2}\right) Y_{t}} & \rho \sqrt{Y_{t}} \\
0 & \sigma Y_{t}^{\beta}
\end{array}\right] d\left[\begin{array}{l}
W_{1}^{P}(t) \\
W_{2}^{P}(t)
\end{array}\right] .
\end{aligned}
$$

(3) Parameter Sensitivity Analysis of Options Pricing. In order to inspect reliance of options pricing effect on accuracy of parameter estimation, we, respectively, analyze sensitivity of each parameter of the model for options price estimation value.

From Figures $4-8$, it is easy to see the parameters monotonicity of options price. Parameters $\kappa$ and $\gamma$ have positive correlation with estimations of options price, and overestimation of parameters will result in overestimation of options price; parameters $\lambda$ and $\sigma$ have negative correlation with estimations of options price, and overestimation of 

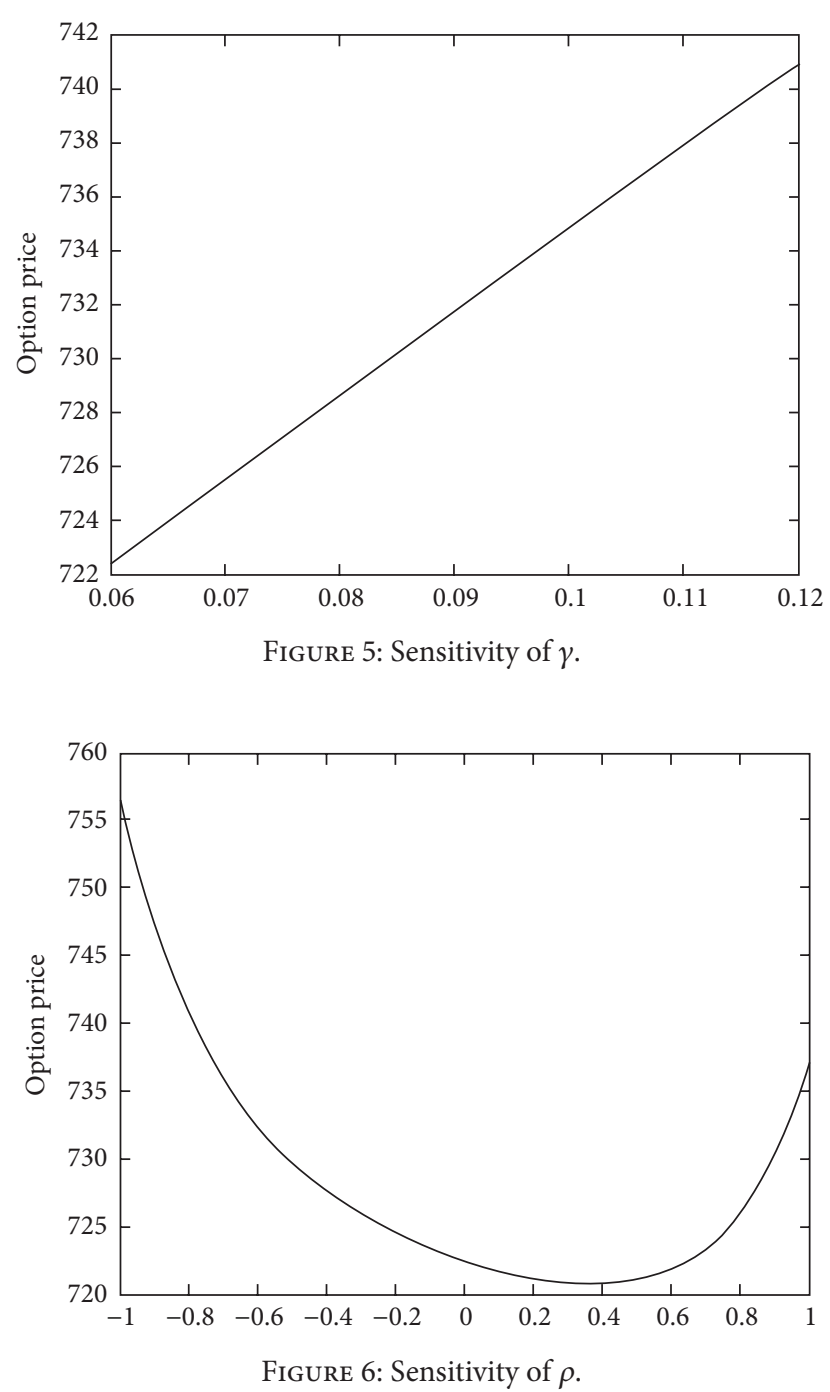

TABLE 4: Analysis of sensitivity of options price to parameters.

\begin{tabular}{lcc}
\hline Parameters & $\begin{array}{c}\text { Parameters estimation } \\
\text { value }\end{array}$ & $\begin{array}{c}\text { Sensitivity of options price } \\
\text { to parameters }\end{array}$ \\
\hline$\kappa$ & 0.4264 & 0.0264 \\
$\gamma$ & 0.0946 & 0.0398 \\
$\rho$ & -0.6284 & 0.0259 \\
$\lambda$ & 3.4543 & 0.1089 \\
$\sigma$ & 0.4822 & 0.0551 \\
\hline
\end{tabular}

parameters will result in underestimation of options price. In particular, influence of correlation coefficient $\rho$ on estimation of options price differs in different ranges of $\rho$; when $\rho$ is less than 0.4 , overestimation of $\rho$ will result in underestimation of options price. When $\rho$ is greater than 0.4 , the result is opposite.

Calculate point elasticity of at-the-money options of parameters estimation, representing sensitivity of options price to each parameter shown in Table 4.

Point elasticities of at-the-money options of parameters estimation remain between 0.02 and 0.11 , which indicate that
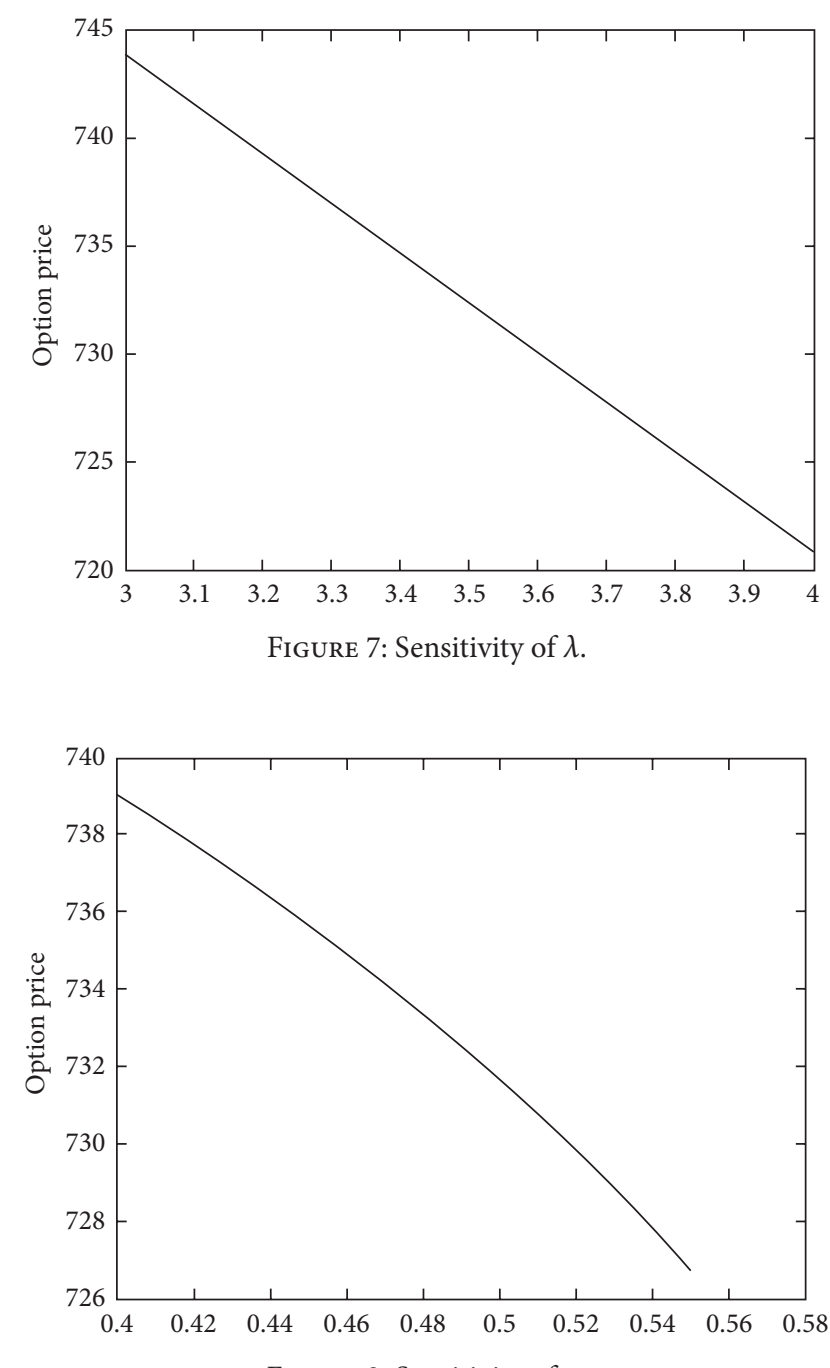

Figure 8: Sensitivity of $\sigma$.

sensitivities of options price to parameters are small and that the influences of errors on options price are not great.

\section{Conclusion}

This paper applies MLE to Heston model and conducts an empirical analysis with market price data of Hong Kong Hang Seng Index Options, and results show that Heston model has a better pricing effect on Hang Seng Index Options. Compared with other options, this model has presented the least pricing error of at-the-money options. Also, we find out that market characteristics have changed in Hong Kong market within two-year period. The parameters estimation effect obviously decreases using two-year market data, compared with estimation effect of about one-year data. This means sample length of MLE should not be too long. And parameters sensitivity analysis of option price indicates that point elasticities of at-the-money options remain between 0.02 and 0.11 , which shows that options price is not that sensitive to parameters and reflects better applicability of MLE. 


\section{Competing Interests}

The authors declare that they have no competing interests.

\section{Acknowledgments}

This paper has obtained funding support from Natural Science Foundation of China (11301560) and Planned Funds for Humanistic and Social Science Research of Ministry of Education (14YJA790048).

\section{References}

[1] J. C. Cox and S. A. Ross, "The valuation of options for alternative stochastic processes," Journal of Financial Economics, vol. 3, no. 1-2, pp. 145-166, 1976.

[2] J. C. Hull and A. White, "The pricing of options on assets with stochastic volatilities," The Journal of Finance, vol. 42, no. 2, pp. 281-300, 1987.

[3] L. O. Scott, "Pricing stock options in a jump-diffusion model with stochastic volatility and interest rates: applications of fourier inversion methods," Mathematical Finance, vol. 7, no. 4, pp. 413-426, 1997.

[4] E. M. Stein and J. C. Stein, "Stock price distributions with stochastic volatility: an analytic approach," Review of Financial Studies, vol. 4, no. 4, pp. 727-752, 1991.

[5] S. L. Heston, "A closed-form solution for options with stochastic volatility with applications to bond and currency options," The Review of Financial Studies, vol. 6, no. 2, pp. 327-343, 1993.

[6] D. S. Bates, "Jumps and stochastic volatility: exchange rate processes implicit in Deutsche mark options," The Review of Financial Studies, vol. 9, no. 1, pp. 69-107, 1996.

[7] G. Bakshi, C. Charles, and Z. Chen, "Empirical performance of alternative option pricing models," The Journal of Finance, vol. 52, no. 5, pp. 2003-2049, 1997.

[8] P. Carr and L. Wu, "The finite moment log stable process and option pricing," The Journal of Finance, vol. 58, no. 2, pp. 753777, 2003.

[9] R. Ahlip and M. Rutkowski, "Pricing of foreign exchange options under the Heston stochastic volatility model and CIR interest rates," Quantitative Finance, vol. 13, no. 6, pp. 955-966, 2013.

[10] K. S. Leung, "An analytic pricing formula for lookback options under stochastic volatility," Applied Mathematics Letters, vol. 26, no. 1, pp. 145-149, 2013.

[11] B. Dupire, "Pricing with a smile," Risk, vol. 7, pp. 18-20, 1994.

[12] I. V. Basawa and B. L. S. Prakasa Rao, Statistical Inference for Stochastic Processes, Academic Press, London, UK, 1980.

[13] E. Jacquier, N. G. Polson, and P. E. Rossi, "Bayesian analysis of stochastic volatility models with fat-tails and correlated errors," Journal of Econometrics, vol. 122, no. 1, pp. 185-212, 2004.

[14] P. Christoffersen and K. Jacobs, "The importance of the loss function in option valuation," Journal of Financial Economics, vol. 72, no. 2, pp. 291-318, 2004.

[15] E. Ruiz, "Quasi-maximum likelihood estimation of stochastic volatility models," Journal of Econometrics, vol. 63, no. 1, pp. 289-306, 1994.

[16] Y. Ait-Sahalia, "Maximum likelihood estimation of discretely sampled diffusions: a closed-form approximation approach," Econometrica, vol. 70, no. 1, pp. 223-262, 2002.
[17] Y. Aït-Sahalia and R. Kimmel, "Maximum likelihood estimation of stochastic volatility models," Journal of Financial Economics, vol. 83, no. 2, pp. 413-452, 2007. 


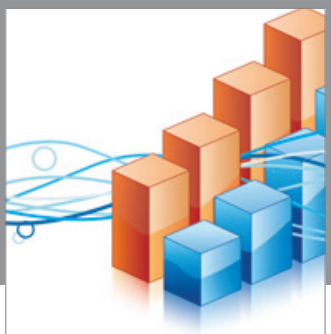

Advances in

Operations Research

vatem alat4

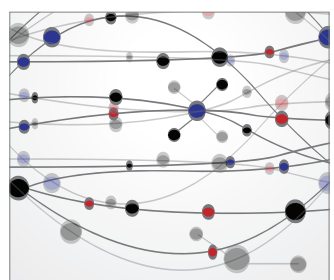

\section{The Scientific} World Journal
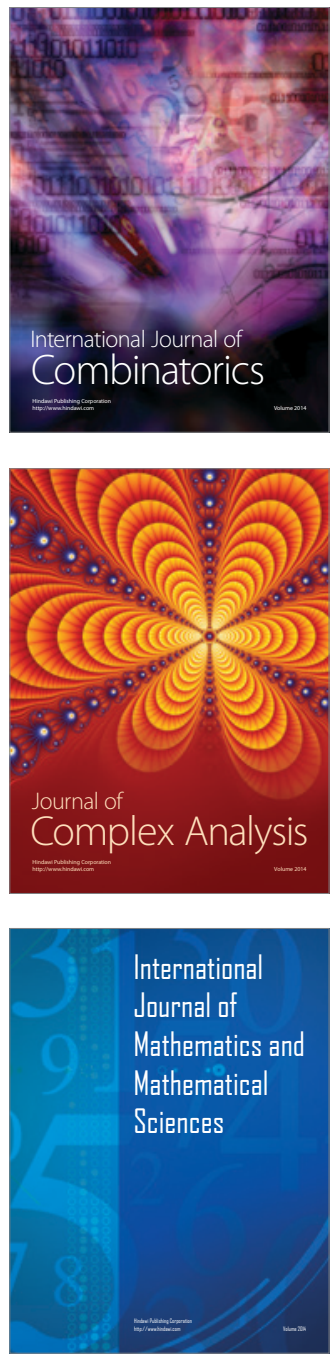
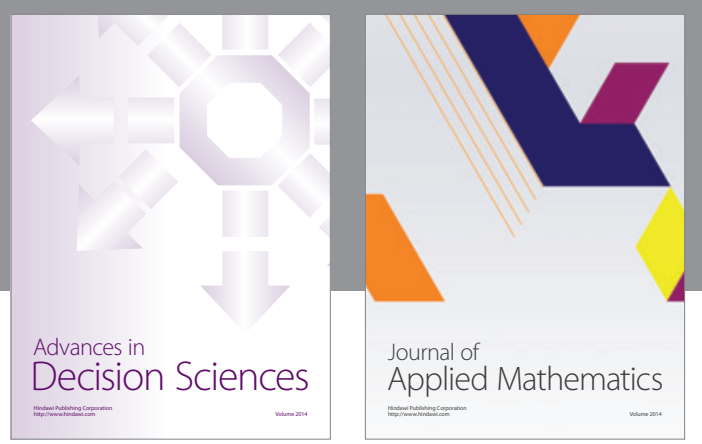

Algebra

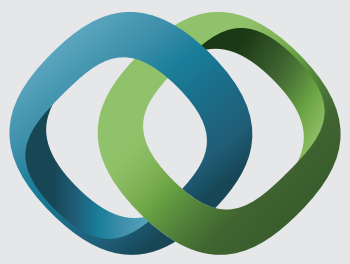

\section{Hindawi}

Submit your manuscripts at

http://www.hindawi.com
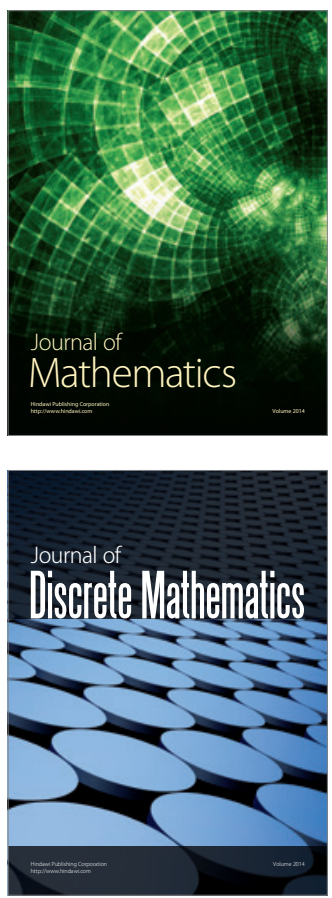

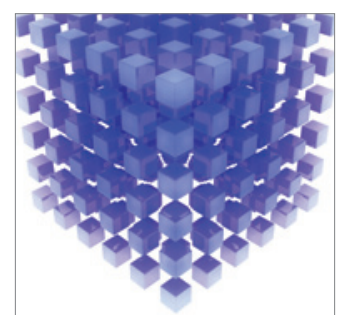

Mathematical Problems in Engineering
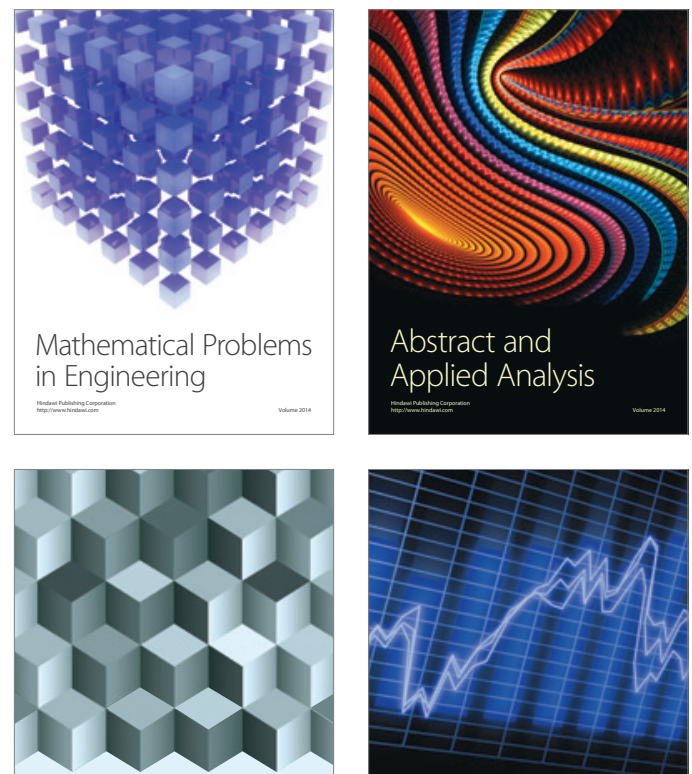

Journal of

Function Spaces

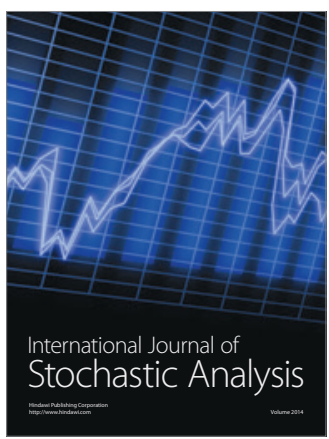

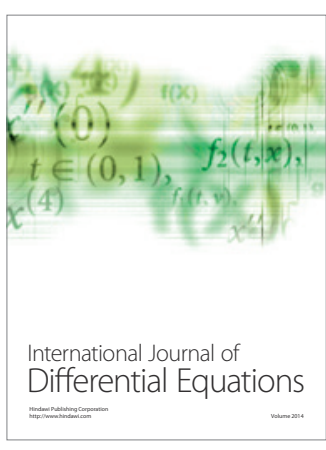
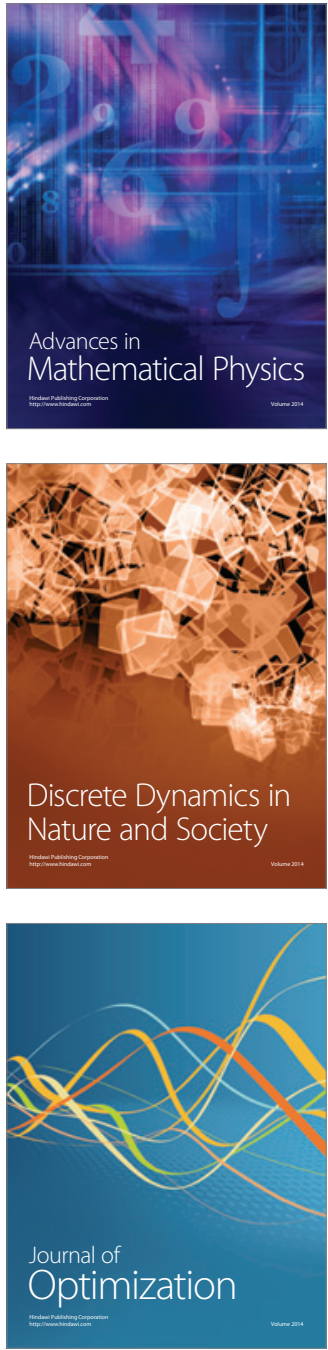